\title{
The Weighted Bergman Kernel and the Green's Function
}

\author{
Steven Krantz ${ }^{1}$ • Pawel M. Wójcicki² ${ }^{(D}$
}

Received: 6 June 2016 / Accepted: 14 September 2016 / Published online: 16 December 2016 (C) The Author(s) 2016. This article is published with open access at Springerlink.com

\begin{abstract}
We study the connection between the weighted Bergman kernel and the Green's function on a domain $W \subset \mathbb{C}$ for which the Green's function exists.
\end{abstract}

Keywords Weighted Bergman kernel · Green's function · Harmonic function

Mathematics Subject Classification Primary 32A36; Secondary 32A25

\section{Introduction}

The Bergman kernel (see for instance $[1,7-9,14,18]$ ) has become a very important tool in geometric function theory, both in one and several complex variables. It turns out that not only the classical Bergman kernel, but also the weighted one can be useful (see $[4,5,11]$ for applications in quantum field theory). Let $W \subset \mathbb{C}$ be a domain (i.e., a connected, open set) such that the Bergman space $L_{H}^{2}(W)$ is a non-zero space and $G_{W}$ the classical Green's function of $W$ (let us recall that $G_{W}$ exists if $\mathbb{C} \backslash W$ is not polar, and this is only if $L_{H}^{2}(W) \neq 0$-see $\left.[3,10]\right)$.

Communicated by Scott McCullough.

Paweł M. Wójcicki

p.wojcicki@mini.pw.edu.pl

Steven Krantz

sk@math.wustl.edu

1 Department of Mathematics, Washington University in St. Louis, St. Louis, MO 63130, USA

2 Faculty of Mathematics and Information Science, Warsaw University of Technology, Koszykowa 75, 00-662 Warsaw, Poland 
It is known, in the classical case, that

$$
K_{W}(z, w)=-\frac{2}{\pi} \frac{\partial^{2}}{\partial z \partial \bar{w}} G_{W}(z, w)
$$

(see [19]) for $z, w \in W, z \neq w$ (it was originally proved in [2] with additional assumptions on $\partial W)$. On the other hand, if $\partial W$ consists of a finite number of Jordan curves, $\rho(z)$ is a positive continuously differentiable function of $x$ and $y$ on a neighborhood of $\bar{W}, K_{W, \rho}(z, w)$ a weighted Bergman kernel of the space $L_{H}^{2}(W, \rho)$ and $G_{W, \rho}$ the Green's function for an operator $P_{\rho}=\frac{\partial}{\partial \bar{z}} \frac{1}{\rho(z)} \frac{\partial}{\partial z}$, then

$$
K_{W, \rho}(z, w)=-\frac{2}{\pi \rho(z) \rho(w)} \frac{\partial^{2}}{\partial z \partial \bar{w}} G_{W, \rho}(z, w)
$$

(see [6]). A very natural question is: "can we relax the regularity of $\partial W$ ?". In this paper we prove that the connection above holds for any domain $W \subset \mathbb{C}$, for which $L_{H}^{2}(W) \neq 0$ (any bounded domain share this property) and for weights $\rho$ such that $\log \rho$ is harmonic. The generalization is not straightforward, since we need to find the connection between a classical Green function and $G_{W, \rho}$. We shall begin with the definitions and basic facts used in this paper. Additionally, because we are dealing with the weighted Bergman kernels, we will recall for which weights in general the weighted Bergman kernel exists (although we are working here with differentiable weights only).

\section{Definitions and Notation}

Let $W \subset \mathbb{C}$ be a domain, and let $\mathcal{W}(W)$ be the set of weights on $W$, i.e., $\mathcal{W}(W)$ is the set of all Lebesque measurable, real-valued, positive functions $\mu$ on $W$ (we consider two weights as equivalent if they are equal almost everywhere with respect to the Lebesque measure on $W)$. For $\mu \in \mathcal{W}(W)$ we denote by $L^{2}(W, \mu)$ the space of all Lebesque measurable, complex-valued, $\mu$-square integrable functions on $W$, equipped with the norm $\|\cdot\|_{W, \mu}:=\|\cdot\|_{\mu}$ and given by the scalar product

$$
\langle f \mid g\rangle_{\mu}:=\int_{W} f(z) \overline{g(z)} \mu(z) d V, \quad f, g \in L^{2}(W, \mu)
$$

The space $L_{H}^{2}(W, \mu)=\mathcal{O}(W) \cap L^{2}(W, \mu)$ is called the weighted Bergman space, where $\mathcal{O}(W)$ denotes the space of all holomorphic functions on the domain $W$. For any $z \in W$ we define the evaluation functional $E_{z}$ on $L_{H}^{2}(W, \mu)$ by the formula

$$
E_{z} f:=f(z), \quad f \in L_{H}^{2}(W, \mu) .
$$

Let us recall the definition (Definition 2.1) of admissible weight given in [13]. 
Definition 2.1 (Admissible weight) A weight $\mu \in \mathcal{W}(W)$ is called an admissible weight, an a-weight for short, if $L_{H}^{2}(W, \mu)$ is a closed subspace of $L^{2}(W, \mu)$ and for any $z \in W$, the evaluation functional $E_{z}$ is continuous on $L_{H}^{2}(W, \mu)$. The set of all a-weights on $W$ will be denoted by $\mathcal{A} \mathcal{W}(W)$.

The definition of admissible weight provides us with existence and uniqueness of the related Bergman kernel and completeness of the space $L_{H}^{2}(W, \mu)$. The concept of a-weight was introduced in [12], and in [13] several theorems concerning admissible weights are proved. An illustrative result is:

Theorem 2.2 [13, Corollary 3.1] Let $\mu \in \mathcal{W}(W)$. If the function $\mu^{-a}$ is locally integrable on $W$ for some $a>0$ then $\mu \in \mathcal{A} \mathcal{W}(W)$.

Now, let us fix a point $t \in W$ and minimize the norm $\|f\|_{\mu}$ in the class $E_{t}=\{f \in$ $\left.L_{H}^{2}(W, \mu) ; f(t)=1\right\}$. It can be proved, in a fashion similar to the classical case, that if $\mu$ is an admissible weight then there exists exactly one function minimizing the norm. Let us denote it by $\phi_{\mu}(z, t)$. The weighted Bergman kernel function $K_{W, \mu}$ is defined as follows:

$$
K_{W, \mu}(z, t)=\frac{\phi_{\mu}(z, t)}{\left\|\phi_{\mu}\right\|_{\mu}^{2}} .
$$

\section{From the Unweighted to the Weighted Case}

Let us recall that we are working with a domain $W \subset \mathbb{C}$, for which $L_{H}^{2}(W) \neq 0$ (any bounded domain has this property). We define the Green's function $G_{W, \rho}$ as the limit in $C^{2}(W)$ of the sequence $\left\{G_{W_{j}, \rho_{j}}\right\}_{j=0}^{\infty}$, for an arbitrary exhaustion $\left\{W_{j}\right\}$ of $W$ by domains with the boundary consisting of a finite number of smooth Jordan curves, and for $\rho_{j}=\rho_{\mid W_{j}}$. We are assuming here that the limit exists and is independent of the exhaustion. Now we will use the result from [19] to prove the following

Theorem 3.1 If $\rho(z)=|\mu(z)|^{2}$, where $\mu \in \mathcal{O}(\bar{W})$, and has no zeros on $\bar{W}$, then

$$
K_{W, \rho}(z, w)=-\frac{2}{\pi \rho(z) \rho(w)} \frac{\partial^{2}}{\partial z \partial \bar{w}} G_{W, \rho}(z, w) .
$$

Proof It is well known that any domain $W \subset \mathbb{C}$ may be written as

$$
W=\bigcup_{j=1}^{\infty} W_{j}, \quad W_{1} \Subset W_{2} \Subset W_{3} \Subset \ldots,
$$

where $\partial W_{j}$ consists of a finite number of smooth Jordan curves (we do not assume any regularity of $\partial W$ ), for any $j \in \mathbb{N}$. Let $\rho_{j}(z)=\left|\mu_{j}(z)\right|^{2}$ where $\mu_{j}=\mu_{\mid W_{j}}$. One can find in ([6], p. 494) that

$$
G_{W_{j}, \rho_{j}}(z, w)=\overline{\mu_{j}(z)} \mu_{j}(w) G_{W_{j}}(z, w),
$$


which in the limit $j \rightarrow \infty$ yields

$$
G_{W, \rho}(z, w)=\overline{\mu(z)} \mu(w) G_{W}(z, w)
$$

(it is a standard fact that $G_{W_{j}}$ converges in $C^{2}(W)$ to $G_{W}$ ). Moreover (by a standard calculation)

$$
\frac{\partial^{2} G_{W, \rho}(z, w)}{\partial z \partial \bar{w}}=\overline{\mu(z)} \mu(w) \frac{\partial^{2} G_{W}(z, w)}{\partial z \partial \bar{w}} .
$$

Let us note that $L_{H}^{2}\left(W,|\mu|^{2}\right)=L_{H}^{2}(W) \neq 0$. Multiplication by $\mu$ is a unitary map from the $L_{H}^{2}\left(W,|\mu|^{2}\right)$ onto $L_{H}^{2}(W)$, so

$$
\mu(z) \overline{\mu(w)} K_{W, \rho}(z, w)=K_{W}(z, w)
$$

because the image of a reproducing kernel by a unitary map is the corresponding reproducing kernel in the target space (this may be also seen by using the complete orthonormal system $\left\{\varphi_{k}(\cdot)\right\}$ in $L_{H}^{2}(W)$ to express $K_{W}$ and $\left\{\varphi_{k}(\cdot) / \mu(\cdot)\right\}$ in $L_{H}^{2}\left(W,|\mu|^{2}\right)$ to express $\left.K_{W, \rho}(z, w)\right)$. Now Theorem 3.1 follows from the result in [19].

\subsection{Non-Holomorphic Weights}

On closer scrutiny, the crucial thing in the proof of Theorem 3.1 was to relate the weighted Green's function to the unweighted one. However, that was possible since holomorphicity of $\mu$ allowed us to find a "bridge" between Green's functions. This relationship turns out to be preserved even if we relax the assumption about holomorphicity of the weight. We will do some reduction which transforms the problem to solving some PDE. It turns out to be possible if only $\log \rho$ is harmonic, as the following reveals.

Theorem 3.2 If $\rho(z)=|\mu(z)|^{2}$, where $\log \rho$ is harmonic on a neighborhood of $\bar{W}$ (and $\mu$ has no zeros on $\bar{W}$ ) then

$$
K_{W, \rho}(z, w)=-\frac{2}{\pi \rho(z) \rho(w)} \frac{\partial^{2}}{\partial z \partial \bar{w}} G_{W, \rho}(z, w) .
$$

Proof Let $\left\{W_{j}\right\}_{j=1}^{\infty}$ be an exhaustion of $W$ and $\rho_{j}=\left|\mu_{j}\right|^{2}$ where $\mu_{j}=\mu_{\mid W_{j}}$. The crucial thing is to find $g_{j}(z)$ such that $u_{j}(w)=g_{j}(w) U_{j}(w)$ is a general solution of the equation

$$
\frac{\partial}{\partial \bar{w}} \frac{1}{\rho_{j}(w)} \frac{\partial}{\partial w} u_{j}(w)=0
$$


and $U_{j}(w)$ is (an arbitrary) complex and harmonic function on $W_{j}$ (we define $g$ on the same way by means of $P_{\rho}$ ). Thus

$$
\begin{aligned}
0= & \frac{\partial}{\partial \bar{w}} \frac{1}{\rho_{j}(w)} \frac{\partial}{\partial w} u_{j}(w)=\frac{\partial}{\partial \bar{w}} \frac{1}{\mu_{j} \overline{\mu_{j}}} \frac{\partial}{\partial w}\left(g_{j}(w) U_{j}(w)\right) \\
= & \frac{\partial}{\partial \bar{w}}\left(\frac{1}{\mu_{j} \overline{\mu_{j}}} \frac{\partial g_{j}}{\partial w} U_{j}\right)+\frac{\partial}{\partial \bar{w}}\left(\frac{1}{\mu_{j} \overline{\mu_{j}}} g_{j} \frac{\partial U_{j}}{\partial w}\right) \\
= & \left(\frac{\partial}{\partial \bar{w}} \frac{1}{\mu_{j} \overline{\mu_{j}}}\right) \frac{\partial g_{j}}{\partial w} U_{j}+\frac{1}{\mu_{j} \overline{\mu_{j}}}\left(\frac{\partial^{2} g_{j}}{\partial \bar{w} \partial w} U_{j}+\frac{\partial g_{j}}{\partial w} \frac{\partial U_{j}}{\partial \bar{w}}\right)+\left(\frac{\partial}{\partial \bar{w}} \frac{1}{\mu_{j} \overline{\mu_{j}}}\right) g_{j} \frac{\partial U_{j}}{\partial w} \\
& +\frac{1}{\mu_{j} \overline{\mu_{j}}}(\frac{\partial g_{j}}{\partial \bar{w}} \frac{\partial U_{j}}{\partial w}+g_{j} \underbrace{\frac{\partial^{2} U_{j}}{\partial \bar{w} \partial w}}_{0})
\end{aligned}
$$

Thus

$$
\left\{\begin{array}{l}
\left(\frac{\partial}{\partial \bar{w}} \frac{1}{\mu_{j} \overline{\mu_{j}}}\right) \frac{\partial g_{j}}{\partial w}+\frac{1}{\mu_{j} \overline{\mu_{j}}} \frac{\partial^{2} g_{j}}{\partial \bar{w} \partial w}=0 \\
\frac{\partial g_{j}}{\partial w}=0 \\
\left(\frac{\partial}{\partial \bar{w}} \frac{1}{\mu_{j} \overline{\mu_{j}}}\right) g_{j}+\frac{1}{\mu_{j} \overline{\mu_{j}}} \frac{\partial g_{j}}{\partial \bar{w}}=0
\end{array}\right.
$$

Remark 3.3 By the equation above, $g_{j}$ is an antiholomorphic function.

Examining the system above, we see that the first equation is a consequence of the second one. Let us focus on the third one:

$$
\left(\frac{\partial}{\partial \bar{w}} \frac{1}{\mu_{j} \overline{\mu_{j}}}\right) g_{j}+\frac{1}{\mu_{j} \overline{\mu_{j}}} \frac{\partial g_{j}}{\partial \bar{w}}=0 .
$$

It may be written in the form

$$
\frac{1}{g_{j}} \frac{\partial g_{j}}{\partial \bar{w}}=\frac{1}{\mu_{j} \overline{\mu_{j}}} \frac{\partial}{\partial \bar{w}}\left(\mu_{j} \overline{\mu_{j}}\right)
$$

Thus, for a given $\mu_{j}$, there is a function $g_{j}$ which must satisfy:

$$
\left\{\begin{array}{l}
\frac{1}{g_{j}} \frac{\partial g_{j}}{\partial \bar{w}}=\frac{1}{\mu_{j} \overline{\mu_{j}}} \frac{\partial}{\partial \bar{w}}\left(\mu_{j} \overline{\mu_{j}}\right) \\
\frac{\partial g_{j}}{\partial w}=0
\end{array}\right.
$$

Notice that, if $\mu_{j}$ is holomorphic and $g_{j}=\overline{\mu_{j}}$, then the system above is satisfied (in this case we get the result of [6]). We may proceed to get the exact form of $g_{j}(z)$, namely: 


$$
\begin{aligned}
\frac{\partial}{\partial \bar{w}} \log g_{j} & =\frac{\partial}{\partial \bar{w}} \log \left(\mu_{j} \overline{\mu_{j}}\right), \\
\log g_{j} & =\log \left(\mu_{j} \overline{\mu_{j}}\right)+h_{j}(w), \\
g_{j}(w) & =\mu_{j} \overline{\mu_{j}} e^{h_{j}(w)}=\left|\mu_{j}(w)\right|^{2} e^{h_{j}(w)},
\end{aligned}
$$

where $h_{j} \in \mathcal{O}\left(\overline{W_{j}}\right)$. Since the $g_{j}$ need to be antiholomorphic, $h_{j}$ is not an arbitrary holomorphic function. Let us proceed to get the exact form of $h_{j}$.

$$
\begin{aligned}
& 0=\frac{\partial g_{j}}{\partial w}=\frac{\partial}{\partial w}\left(\mu_{j} \overline{\mu_{j}}\right) e^{h_{j}}+\mu_{j} \overline{\mu_{j}} e^{h_{j}} \frac{\partial h_{j}}{\partial w}, \\
& 0=\frac{\partial}{\partial w}\left(\mu_{j} \overline{\mu_{j}}\right)+\mu_{j} \overline{\mu_{j}} \frac{\partial h_{j}}{\partial w} .
\end{aligned}
$$

So

$$
h_{j}(w)=-\log \left|\mu_{j}\right|^{2}+l_{j}(w)
$$

where $l_{j}$ is antiholomorphic. Taking $\frac{\partial^{2}}{\partial w \partial \bar{w}}$ we see that $\log \left|\mu_{j}\right|$ must be harmonic. Take $l_{j}(w)=\overline{h_{j}(w)}+\log \left|\mu_{j}\right|^{2}+2 i \operatorname{Im} h_{j}(w)$. So

$$
\left.0=\frac{\partial}{\partial w}\left(\overline{h_{j}(w)}+\log \left|\mu_{j}\right|^{2}+2 i \operatorname{Im} h_{j}(w)\right)=\frac{\partial}{\partial w} \log \left|\mu_{j}\right|^{2}+2 i \frac{\partial}{\partial w} \operatorname{Im} h_{j}(w)\right)
$$

By $\frac{\partial}{\partial w}=\frac{1}{2}\left(\frac{\partial}{\partial x}-i \frac{\partial}{\partial y}\right)$ we see that

$$
\left\{\begin{array}{r}
\frac{1}{2} \frac{\partial}{\partial x} \log \left|\mu_{j}\right|^{2}+\frac{\partial}{\partial y} \operatorname{Im} h_{j}(w)=0 \\
-\frac{1}{2} \frac{\partial}{\partial y} \log \left|\mu_{j}\right|^{2}+\frac{\partial}{\partial x} \operatorname{Im} h_{j}(w)=0
\end{array}\right.
$$

Write $\int \log \left|\mu_{j}(w)\right| d y=F_{j}(x, y)+c(x)$, where $w=x+i y$ and $\frac{\partial}{\partial y} F_{j}(x, y)=$ $\log \left|\mu_{j}(w)\right|^{2}$. Since $\log \left|\mu_{j}\right|^{2}$ is harmonic, we have

$$
\frac{\partial}{\partial y} \Delta F_{j}=0
$$

or just $\triangle F_{j}=d_{j}(x)$, where $d_{j}$ is some differentiable function of $x$ only. Thus

$$
\operatorname{Im} h_{j}(w)=-\frac{1}{2} \frac{\partial}{\partial x} F_{j}+\frac{1}{2} e_{j}(x)
$$

where $\int d_{j}(x) d x=e_{j}(x)+c(y)$. We may easily check that $\operatorname{Im} h_{j}(w)$ is harmonic. To this end,

$$
h_{j}(w)=-\frac{1}{2} \log \left|\mu_{j}\right|^{2}+i\left(-\frac{1}{2} \frac{\partial}{\partial x} F_{j}+\frac{1}{2} e_{j}(x)\right)
$$


where $\frac{\partial}{\partial y} F_{j}(x, y)=\log \left|\mu_{j}(w)\right|^{2}$, and $e_{j}^{\prime}(x)=d_{j}(x)$.

Thus

$$
g_{j}(z)=\left|\mu_{j}(z)\right|^{2} e^{h_{j}(z)}
$$

where $h_{j}$ is given above. Now, by the definition of $\mu_{j}$ we have that $g_{j} \rightarrow g=$ $|\mu(z)|^{2} e^{h(z)}$, where

$$
h(w)=-\frac{1}{2} \log |\mu|^{2}+i\left(-\frac{1}{2} \frac{\partial}{\partial x} F+\frac{1}{2} e(x)\right)
$$

and by Harnack's theorem $\log |\mu|$ is harmonic.

Again (as in [6])

$$
G_{W_{j}, \rho_{j}}(z, w)=g_{j}(z) \overline{g_{j}(w)} G_{W_{j}}(z, w),
$$

so

$$
\frac{\partial^{2} G_{W_{j}, \rho_{j}}(z, w)}{\partial z \partial \bar{w}}=g_{j}(z) \overline{g_{j}(w)} \frac{\partial^{2} G_{W_{j}}(z, w)}{\partial z \partial \bar{w}} .
$$

By the regularity of any $\partial W_{j}$ we have

$$
K_{W_{j}, \rho_{j}}(z, w)=-\frac{2}{\pi \rho_{j}(z) \rho_{j}(w)} \frac{\partial^{2}}{\partial z \partial \bar{w}} G_{W_{j}, \rho_{j}}(z, w),
$$

which in the limit as $j \rightarrow \infty$ yields

$$
\begin{aligned}
K_{W, \rho}(z, w) & =-\frac{2}{\pi \rho(z) \rho(w)} \overline{g(z)} g(w) \frac{\partial^{2}}{\partial z \partial \bar{w}} G_{W}(z, w) \\
& =-\frac{2}{\pi \rho(z) \rho(w)} \frac{\partial^{2} G_{W, \rho}(z, w)}{\partial z \partial \bar{w}} .
\end{aligned}
$$

(we made use of the result in [20] for the LHS of the above).

\section{Remarks and Some Applications}

One could try the "reduction" used in the proof above to find a connection between the classical Green's function and the Green's function of some other differential operator of elliptic type.

It is well established that weighted Bergman spaces are both intrinsically interesting and a powerful analytic tool. Our purpose in this paper has been to develop this set of ideas, and particularly the connection between the Bergman kernel and the Green's function in the weighted context. Some of the applications might be: 
(a) With the established connection between weighted Bergman kernel and Green's function in hand, we can reformulate the weighted version of the so called "small conjecture" (Is the so called Skwarczyński distance equivalent to the Bergman distance?—see $[16,17])$ as:

Remark 4.1 Assume $W \Subset \mathbb{C}$, and $\mu$ is a continuously differentiable function of $x$ and $y$ on a neighborhood of $\bar{W}$. Then $t_{n} \rightarrow t \in \partial W$ represents defective evaluation (see [17]) iff

$$
-\frac{2}{\pi \rho(z) \rho(w)} \frac{\partial^{2}}{\partial z \partial \bar{w}} G_{W, \rho}(z, w)\left(\cdot, t_{n}\right) \rightarrow \gamma
$$

weakly in $L_{H}^{2}(W, \mu)$ and

$$
-\frac{2}{\pi \rho(z) \rho(w)} \frac{\partial^{2}}{\partial z \partial \bar{w}} G_{W, \rho}(z, w)\left(t_{n}, t_{n}\right) \rightarrow \kappa^{2}
$$

where $\|\gamma\| \neq \kappa$. This is important, since the involved so-called Skwarczyński distance is biholomorphically invariant, and given more explicitly than the Bergman distance.

(b) Using the method of alternating projections (see [15]), we can recover (having some Dirichlet and Neuman boundary conditions on $\left.G_{W}\right) G_{W, \mu}$ for an arbitrary domain $W$ lying in $\mathbb{C}$.

Acknowledgements We would like to thank the Referee for very valuable comments and suggestions regarding this paper, especially the proof of Theorem 3.1.

Open Access This article is distributed under the terms of the Creative Commons Attribution 4.0 International License (http://creativecommons.org/licenses/by/4.0/), which permits unrestricted use, distribution, and reproduction in any medium, provided you give appropriate credit to the original author(s) and the source, provide a link to the Creative Commons license, and indicate if changes were made.

\section{References}

1. Bergman, S.: The kernel function and conformal mappings, 2nd edn. American Mathematical Society, Providence, RI (1970)

2. Bergman, S., Schiffer, M.: Kernel functions and conformal mapping. Compos. Math. 8, 205-249(1951)

3. Carleson, L.: Selected problems on exceptional sets. Van Nostrand, Princeton (1967)

4. Engliš, M.: Toeplitz operators and weighted Bergman kernels. J. Funct. Anal. 255(6), 1419-1457 (2008)

5. Engliš, M.: Weighted Bergman kernels and quantization. Comm. Math. Phys. 227(2), 211-241 (2002)

6. Garabedian, P.R.: A partial differential equation arising in conformal mapping. Pacific J. Math. 1, 485-524 (1951)

7. Jarnicki, M., Pflug, P.: Invariant distances and metrics in complex analysis, 2nd edn. de Gruyter Expositions, Berlin (2013)

8. Krantz, S.G.: Function theory of several complex variables. AMS Chelsea Publishing, Providence, RI (2001)

9. Krantz, S.G.: Geometric analysis of the Bergman Kernel and Metric. Springer, New York (2013)

10. Myrberg, P.: Über die existenz der Greenschen Funktionen auf einer gegeben Riemannschen Fläche. Acta Math. 61, 39-79 (1933) 
11. Odzijewicz, A.: On reproducing kernels and quantization of states. Commun. Math. Phys. 114, 577-597 (1988)

12. Pasternak-Winiarski, Z.: On the dependence of the reproducing kernel on the weight of integration. J. Funct. Anal. 94(1), 110-134 (1990)

13. Pasternak-Winiarski, Z.: On weights which admit the reproducing kernel of Bergman type. Int. J. Math. Math. Sci. 15(1), 1-14 (1992)

14. Shabat, B.: Introduction to complex analysis, Part II: functions of several variables, 2nd edn. American Mathematical Society, Providence, RI (1992)

15. Skwarczynski, M.: Alternating projections in complex analysis. In: Complex analysis and applications (Varna, 1983), pp. 192-199. Publishing House of the Bulgarian Academy of Sciences, Sofia (1985)

16. Skwarczyński, M.: Biholomorphic invariants related to the Bergman function. Warsaw, Polish Scientific Publishing Company: Dissertationes Mathematicae (Rozprawy Matematyczne), vol. 173 (1980)

17. Skwarczyński, M.: Metric completeness in holomorphic geometry. Bull. Soc. Sci. Lett. Łódź Sér. Rech. Déform 51, 11-22 (2006)

18. Skwarczyński, M., Mazur, T.: Wstepne twierdzenia teorii funkcji wielu zmiennych zespolonych (Polish). Krzysztof Biesaga, Warszawa (2001)

19. Suszczyński, A.: O pewnej tożsamości dla funckji Greena i Bergmana (Polish). Zeszyty Naukowe Filii Uniwersytetu Warszawskiego w Bialymstoku. 39, 113-118 (1984)

20. Wójcicki, P.M.: Weighted Bergman kernel function, admissible weights and the Ramadanov theorem. Matematychni Studii. 42(2), 160-164 (2014) 